\title{
The Successful Use of Dual Flow Diverters in the Treatment of a Fusiform Middle Cerebral Artery Aneurysm

\author{
Panduranga Seetahal-Maraj, MRCS ${ }^{1^{*}}$ (D) Narindra Ramnarine, FRCS $^{2}$ and Yourki Pierri \\ Tairouz Zeitoune, $M D^{3}$
}

${ }^{1}$ Department of Neurosurgery, San Fernando General Hospital, San Fernando, Trinidad and Tobago, West Indies

${ }^{2}$ Department of Neurosurgery, San Fernando General Hospital, San Fernando, Trinidad and Tobago, West Indies

${ }_{3}^{3}$ Interventional Radiology, Caribbean Heart Care, St Clair Medical Center, Trinidad and Tobago, West Indies

*Corresponding author: Panduranga Seetahal-Maraj, Department of Neurosurgery, San Fernando General Hospital, San Fernando, 389 Calcutta Road \#2, Freeport, Trinidad, West Indies, Tel: 1868-755-2875

\begin{abstract}
Background and importance: The management of fusiform anterior circulation aneurysms has been controversial, due to the rare nature of such lesions. They provide a unique management challenge compared to saccular aneurysms, and a variety of microsurgical techniques have been attempted in the past, with varying success rates. Fusiform middle cerebral artery aneurysms can be fraught with morbidity and even mortality, if perforator ischaemia results from intervention. In this neuroendovascular era, flow diverters have shown promise in both securing the aneurysm and minimising neurologic deficits associated with treatment.

Clinical presentation: We report on a 29-year-old man who had successful treatment of his ruptured terminal ICA-proximal M2 fusiform aneurysm with flow diverters. Both immediate and 3-month angiograms had no evidence of early or delayed aneurysmal filling (O'Kelly-Marotta grade D). There was no permanent neurologic deficit in this patient, and he resumed full activity within three months.

Conclusion: Dual flow diverter management of fusiform MCA aneurysms are a viable option, and can be added to the surgeon's armamentarium when these lesions are encountered.
\end{abstract}

\section{Keywords}

Flow diverter, Fusiform aneurysm, Middle cerebral artery

\section{Abbreviations}

GCS: Glasgow Coma Scale; MCA: Middle Cerebral Artery; WFNS: World Federation Of Neurosurgical Societies; OKM: O'Kelly-Marrotta; ICA: Internal Carotid Artery; SAH: Subarachnoid Haemorrhage; FRED: Flow Re-Direction Endoluminal Device

\section{Introduction}

The management of fusiform anterior circulation aneurysms has been controversial, due to the rare nature of such lesions. They provide a unique management challenge compared to saccular aneurysms, since they do not possess a conventional aneurysmal neck, and therefore cannot be clipped. A number of microsurgical techniques have been attempted in the past, including parent vessel reconstruction, aneurysmal wrapping and bypass. Success rates have been variable, and no consensus has been reached on the ideal management strategy.

In particular, fusiform middle cerebral artery (MCA) aneurysms can be fraught with morbidity and mortality, if there is perforator ischaemia secondary to intervention. However, in this neuroendovascular era, flow diverters have shown promise in both securing the fusiform aneurysm and minimising neurologic deficits associated with treatment. This has been reported with posterior circulation aneurysms, and we present a case of a fusiform MCA aneurysm that was successfully secured using dual flow diverters, with excellent neurological outcome.

\section{Case Description}

A 29-year-old male presented with a one-day history of headaches and left upper limb weakness. The headaches were 5/10 in severity and associated

Citation: Maraj PS, Ramnarine N, Zeitoune YPT (2021) The Successful Use of Dual Flow Diverters in the Treatment of a Fusiform Middle Cerebral Artery Aneurysm. Neurosurg Cases Rev 4:077. doi. org/10.23937/2643-4474/1710077

Accepted: September 02, 2021; Published: September 04, 2021

Copyright: (C) 2021 Maraj PS, et al. This is an open-access article distributed under the terms of the Creative Commons Attribution License, which permits unrestricted use, distribution, and reproduction in any medium, provided the original author and source are credited. 
with mild photophobia. He denied any nausea/vomiting and had no fever or seizures. The rest of his history was unremarkable.

On examination, his GCS was $15 / 15$, with both pupils being $3 \mathrm{~mm}$ and equally reactive. There was no evidence of ophthalmoplegia. Muscle tone and bulk in all limbs appeared normal, but he had grade $4 / 5$ power in the left upper limb, to elbow flexion, wrist extension and grip strength. Reflexes were $3+$ in the left upper limb, and $2+$ in the remainder of the limbs. Both plantars were down going, and there was no clonus. His gait was normal.

CTbrain showed an acute subarachnoid haemorrhage, with the blood load distributed mainly in the right sylvian fissure. A large mass was noted in the region of the terminal internal carotid artery (ICA), with a suspicion of a giant aneurysm. CT angiogram was done, and a large fusiform dilation of the terminal ICA-proximal M2 region was noted, in keeping with a ruptured fusiform aneurysm. The patient's subarachnoid haemorrhage (SAH) was graded as Fisher 3, Hunt and Hess grade 3, World Federation of Neurological Surgeons grade 3.

A catheter angiogram was performed (Figure 1), which confirmed the fusiform dilatation of the terminal ICA - proximal M2 segment and delineated the anatomy of the thalamo-perforators that arose from this segment of the middle cerebral artery (MCA).

Optimization of cerebral perfusion was undertaken, maintaining systolic blood pressures between 120-
$160 \mathrm{mmHg}$, and Nimodipine commenced. A decision was made for the use of flow-diverters to treat the fusiform segment. This would allow reconstruction of the parent artery and exclusion of the aneurysmal segment from the cerebral circulation. Importantly, there would be a greater chance of preservation of the thalamo-perforators, compared to using the described microsurgical techniques (wrapping, bypass, etc).

The patient was started on dual anti-platelet therapy (Aspirin and Clopidogrel) 5 days prior to the procedure. A 6 French neuron intermediate guide catheter was placed in the right cavernous segment of the ICA. A 0.027" Marksman Microcatheter was used over a synchro 0.014" microwire to catheterize the superior M2 trunk, and a $3 \mathrm{~mm} \times 25 \mathrm{~mm}$ Pipeline flow diverter placed from proximal $M 2$ to the proximal third of the aneurysm.

The Microcatheter was unable to be navigated over the pusher and had to be removed. An Echelon 10 Microcatheter was shaped to 90 degrees and used to re-enter the flow diverter, then exchanged over a Transcend wire to a Headway 27 Microcatheter. A $4 \mathrm{~mm}$ × $32 \mathrm{~mm}$ FRED (Flow Re-direction Endoluminal Device) flow diverter was placed into the first flow diverter, covering the region up to the terminal ICA.

The immediate post-angiogram showed adequate placement of the flow diverters, with complete exclusion of the aneurysmal segment from the circulation, graded as O'Kelly-Marotta D (OKM), and good filling of the
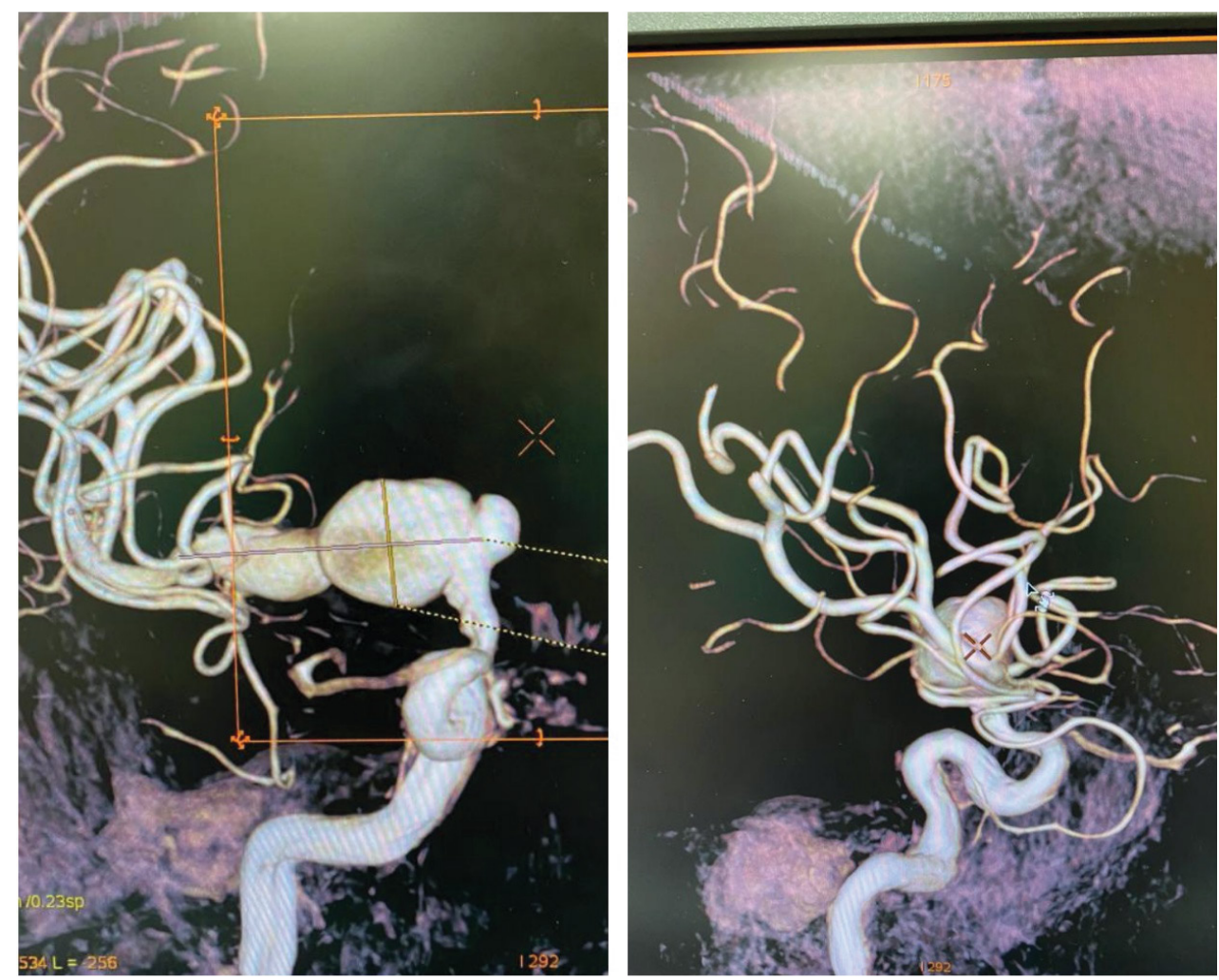

Figure 1: Preoperative catheter angiogram showing right M1 fusiform aneurysm in AP (left) and oblique (right) views. Numerous lenticulo-striate perforators can be seen originating from the fusiform region. 

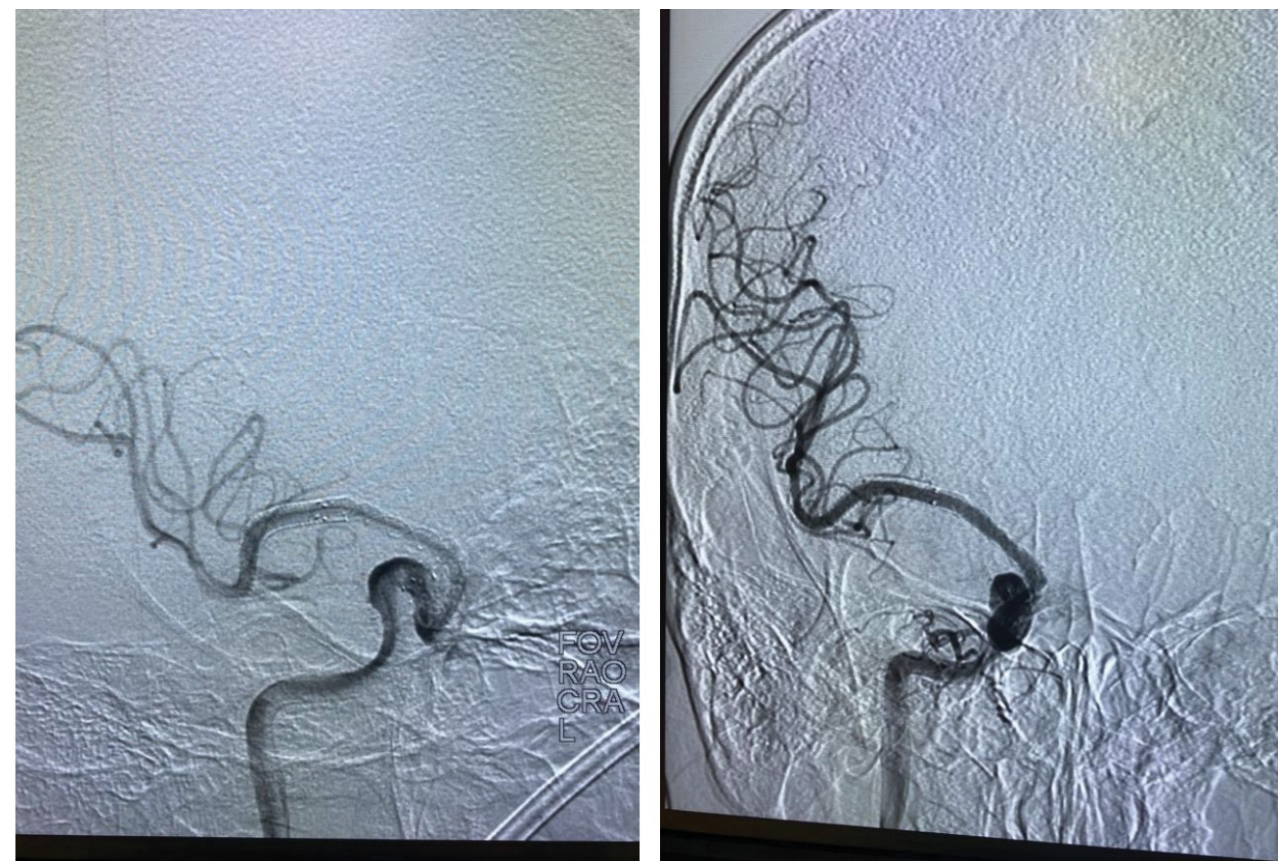

Figure 2: Post-flow diverter placement, showing complete exclusion of aneurysmal segment from circulation in AP (left) and oblique (right) views. Graded as OKM D.

normal vessels. The patient was observed in the ICU overnight and stepped down to the ward within 24 hours.

He remained neurologically stable, and at two weeks post-intervention had improvement in the left upper limb power grade from $4 / 5$ to $4+/ 5$. At three months post-endovascular intervention, his power is now $5 / 5$ in all limbs, and 3-month angiogram (Figure 2) shows no aneurysmal recurrence (OKM grade $D$ ). He remains on dual anti-platelet therapy, which will be continued for a minimum of one year.

\section{Discussion}

Fusiform aneurysms comprise a small subset of intracranial aneurysms and pose unique challenges when compared to saccular aneurysms. They represent between $3-13 \%$ of all intracranial aneurysms, with a predilection for the posterior circulation $[1,2]$. They lack an aneurysmal neck, and involves a pathologically dilated segment of a vessel, with separate inflow and outflow sites [3]. This can be due to dissection, atherosclerosis and collagen-vascular disease [4]. Patients can present with ischemic/mass effect symptoms, including dizziness, ataxia, cranial nerve palsies, hemiparesis, aphasia [2]. Subarachnoid haemorrhage and its sequelae are also observed.

This lack of a true neck renders the standard microsurgical clipping and conventional coiling techniques ineffective [5]. Clip reconstruction, parentvessel sacrifice and complex bypass techniques have all been attempted for fusiform aneurysms of the MCA. These can be technically challenging, with a high risk of ischemic events [6].
However, due to the factors stated above, endovascular management of this subset of aneurysms is growing in popularity. Since the first prospective multi-centre trial of flow diverter (Pipeline) use in management of intracranial aneurysms [7], their role in securing fusiform aneurysms has been explored. They have shown high rates of occlusion at 6 months, and a safety profile similar to stent assisted coiling. Our use of flow diverters in this patient is one of few reported successful cases in the literature.

The concern with flow diverter use, especially in the region of $\mathrm{M} 1$, is of lenticulostriate perforator occlusion and significant ischemic events. Mixed results have been reported in the past, with Lall, et al. reporting on three patients who had ischemic events after placement of a flow diverter [8], but Chalouhi, et al. reporting on the safety of the Pipeline flow diverter in seven patients with no ischemic events [9].

However, Alturki, et al. managed a similar M1 aneurysm in 2018, with sequential flow diverter/ coiling and no clinical or radiological evidence of perforator compromise, and similar excellent outcome to our patient [6]. Further to this, Wagner, et al. in 2019 retrospectively reviewed 52 patients who had flow diverter placement in the MCA, proximal anterior cerebral artery (ACA) and distal ICA, in which the medial and lateral lenticulostriates were covered. Discontinuation of anti-platelet drugs prior to six months was the only factor found to correlate with an increased risk of stroke [10].

The O'Kelly-Marotta grading system is a proposed scale for assessment of aneurysms treated by flow diversion. A grade is assigned based on the degree of 
filling (A-D) and degree of stasis (1-3) observed in the angiographic phases (arterial, capillary, venous). Our patient was Grade D, implying complete exclusion of the aneurysm from the circulation, thereby eliminating the risk of rupture/bleeding [11].

Follow-up of these patients includes angiograms at 3-6 months, one year, then at 1-3 year intervals based on the clinical state of the patient and surgeon preference. Dual antiplatelet therapy is continued for a year, and then aspirin monotherapy indefinitely.

\section{Conclusion}

The management of fusiform MCA aneurysms still has no definite consensus, but in the era of endovascular intervention and flow diverters, excellent results have been obtained, both clinically and radiographically. While data is limited at this time, we have seen promise of flow diverter management of these aneurysms, with an acceptable safety profile and minimal risk of ischemic events. The use of dual flow diverters in the M1 segment was not associated with negative neurologic outcomes or perforator ischemia.

\section{Acknowledgements}

All authors have contributed equally to the creation, drafting and editing of this manuscript. There were no sources of funding, and none of the authors have any financial disclosures.

\section{References}

1. Little JR, St Louis P, Weinstein M, Dohn DF (1981) Giant fusiform aneurysm of the cerebral arteries. Stroke 12: 183188.
2. Barletta EA, Ricci RL, Silva RDG, Gaspar RHM, Araujo JFM, et al. (2018) Fusiform aneurysms: A review from its pathogenesis to treatment options. Surg Neurol Int 9: 189.

3. Anson JA, Lawton MT, Spetzler RF (1996) Characteristics and surgical treatment of dolichoectatic and fusiform aneurysms. J Neurosurg 84: 185-193.

4. Park SH, Yim M Bin, Lee CY, Kim E, Son El (2008) Intracranial fusiform aneurysms: It's pathogenesis, clinical characteristics and managements. J Korean Neurosurg Soc 44: 116-123.

5. Awad AJ, Mascitelli JR, Haroun RR, De Leacy RA, Fifi JT, et al. (2017) Endovascular management of fusiform aneurysms in the posterior circulation: The era of flow diversion. Neurosurg Focus 42: 1-7.

6. Alturki AY, Schmalz PGR, Ogilvy CS, Thomas AJ (2018) Sequential coiling-assisted deployment of flow diverter for treatment of fusiform middle cerebral artery aneurysms. Oper Neurosurg (Hagerstown, Md). 15: E13-E18.

7. Nelson PK, Lylyk P, Szikora I, Wetzel SG, Wanke I, et al. (2011) The pipeline embolization device for the intracranial treatment of aneurysms trial. AJNR Am J Neuroradiol 32: 34-40.

8. Lall RR, Crobeddu E, Lanzino G, Cloft HJ, Kallmes DF (2014) Acute branch occlusion after Pipeline embolization of intracranial aneurysms. J Clin Neurosci 21: 668-672.

9. Chalouhi N, Tjoumakaris S, Dumont AS, Gonzalez LF, Randazzo C, et al. (2013) Treatment of posterior circulation aneurysms with the pipeline embolization device. Neurosurgery 72: 883-889.

10. Wagner KM, Srinivasan VM, Srivatsan A, Ghali MGZ, Thomas AJ, et al. (2019) Outcomes after coverage of lenticulostriate vessels by flow diverters: A multicenter experience. J Neurosurg 132: 473-480.

11. O'kelly CJ, Krings T, Fiorella D, Marotta TR (2010) A novel grading scale for the angiographic assessment of intracranial aneurysms treated using flow diverting stents. Interv Neuroradiol 16: 133-137. 